\title{
Authors' reply: Communicating evidence-based practice in menopause
}

\author{
Susan R. Davis, Nanette Santoro, Irene Lambrinoudaki, Maryann Lumsden,
} Gita D. Mishra, Lubna Pal, Margaret Rees and Tommaso Simoncini

We appreciate the concerns raised by Dr Prior in her correspondence on our Primer (Menopause. Nat. Rev. Dis. Primers 1, $15004(2015))^{1}$, which raised some interesting points (Perimenopause and menopause as oestrogen deficiency while ignoring progesterone. Nat. Rev. Dis. Primers http://dx.doi.org/10.1038/ nrdp.2015.31 (2015)) $)^{2}$.

The selection of menopause as a topic in this journal highlights the importance of this life phase for women. We were required to format our article according to specific journal guidelines, but this should not to be interpreted as 'medicalizing menopause'. Rather, our enthusiasm to communicate evidence-based practice to the readership counterbalanced the concern about casting menopause as a disease state. It is difficult to compose a review that encompasses such a large part of a woman's life and that addresses the shortterm and chronic conditions arising from menopause, while highlighting the nuances between the menopausal transition and the postmenopausal years.

We note the contribution that Dr Prior has made to the literature regarding treatment of vasomotor symptoms with progesterone ${ }^{3}$. However, we do not agree that oestrogen has never been shown to reduce vasomotor symptoms in perimenopausal women. First, the many trials of oestrogen treatment of hot flushes have adhered to the US FDA guidance ${ }^{4}$, which recommends that 'only postmenopausal women be included'. However, the inclusion criterion of 6 months of amenorrhoea accompanied by follicle-stimulating hormone levels of $>40 \mathrm{mIU}$ per ml almost certainly includes women who are perimenopausal. Second, studies on hormonal control of hot flushes in perimenopausal women that have used oestrogen in combination with progestin have demonstrated efficacy against hot flushes specifically in perimenopausal women $^{5,6}$. Low-dose transdermal oestradiol has also been shown to be effective in combination with a levonorgestrel intrauterine system in controlling hot flushes in perimenopausal women ${ }^{7,8}$.

Finally, we do not agree with Dr Prior that the concept of oestrogen deficiency is 'out of date. It may not be perfect, but it is the best explanation we currently have.

Susan R. Davis is at the School of Public Health and Preventive Medicine, Monash University, 99 Commercial Road, Melbourne, Victoria 3004, Australia.

Nanette Santoro is at the University of Colorado School of Medicine, Aurora, Colorado, USA.

Irene Lambrinoudaki is at the Medical School, University of Athens, and Aretaieio University Hospital, Athens,

Greece.
Maryann Lumsden is at the Reproductive \& Maternal Medicine, Glasgow Royal Infirmary, Glasgow, UK.

Gita D. Mishra is at the School of Population Health Faculty of Medicine and Biomedical Sciences, University of Queensland, Queensland, Australia

Lubna Pal is at the Department of Obstetrics, Gynecology \& Reproductive Sciences, Yale University School of Medicine, New Haven, Connecticut, USA.

Margaret Rees is at the Women's Centre, John Radcliffe Hospital, Oxford, UK

Tommaso Simoncini is at the University of Pisa, Pisa, Italy. Correspondence to S.R.D. e-mail:susan.davis@monash.edu

doi: $10.1038 /$ nrdp. 2015.32

1. Davis, S. R. et al. Menopause. Nat. Rev. Dis. Primers 1, 15004 (2015).

2. Prior, J. C. Perimenopause and menopause as oestrogen deficiency while ignoring progesterone. Nat. Rev. Dis. Primers http://dx.doi.org/10.1038/ nrdp. 2015.31 (2015)

3. Hitchcock, C. L. \& Prior, J. C. Oral micronized progesterone for vasomotor symptoms - a placebocontrolled randomized trial in healthy postmenopausal women. Menopause 19, 886-893 (2012).

4. U.S. Department of Health and Human Services. Guidance for Industry. Estrogen and estrogen/ progestin drug products to treat vasomotor symptoms and vulvar and vaginal atrophy symptoms recommendations for clinical evaluation. FDA [online], http://www.fda.gov/downloads/ScienceResearch/ SpecialTopics/WomensHealthResearch/UCM133343. pdf (2003).

5. Shargil, A. A. Hormone replacement therapy in perimenopausal women with a triphasic contraceptive compound: a three-year prospective study. Int. J. Fertil. 30, 15-28 (1985)

6. Casper, R. F., Dodin, S. \& Reid, R. The effect of $20 \mathrm{mcg}$ ethinyl estradiol/1 gm norethindrone acetate (Minestrin $^{\mathrm{TM}}$ ), a low-dose oral contraceptive, on vaginal bleeding patterns, hot flashes, and quality of life in symptomatic periomenopausal women. Menopause 4 , 139-147 (1997).

7. Santoro, N. et al. Use of a levonorgestrel-containing IUS with supplemental estrogen improves symptoms in perimenoapusal women - a pilot study. Menopause (in the press).

8. Hampton, N. R. et al. Levonorgestrel intrauterine system (LNG-IUS) with conjugated oral equine estrogen: a successful regimen for HRT in perimenopausal women. Hum. Reprod. 20 2653-2660 (2005).

Competing interests

S.R.D has received honoraria from Abbott Pharmaceuticals and is an investigator for Trimel Pharmaceuticals and Lawley Pharmaceuticals; all other authors declare no competing interests. 